\title{
Lebertransplantation bei Kindern: Investition fürs Leben
}

\author{
Barbara Wildhaber ${ }^{a}$, Valérie McLin ${ }^{b}$, Dominique Bellic

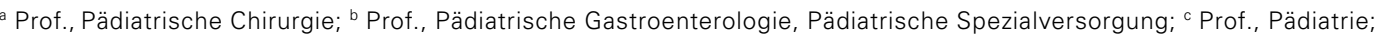 \\ Schweizerisches Zentrum für Kinderleberkrankheiten, Departement für Kinder und Jugendliche, Genfer Universitätsspital (HUG)
}

\begin{abstract}
Die Lebertransplantation bei Kindern ist ein äusserst komplexer medizinisch-chirurgischer Eingriff, der an Personal und Medizintechnik extrem hohe Ansprüche stellt. Bei terminalen Lebererkrankungen von Kindern ist sie heute die Behandlung der Wahl. Dank ihr können junge Patienten, deren Leben bedroht ist, eines Tages aktive Bürger und Eltern werden. Indem wir für ihre Gesundheit sorgen, investieren wir zugleich in unsere Gesellschaft!
\end{abstract}

Die erste Lebertransplantation wurde 1963 in Denver, Colorado, von Professor Thomas Starzl an einem dreijährigen Kind durchgeführt, bei dem eine angeborene Erkrankung der Gallenwege (Gallengang-Atresie) vorlag und das während des Eingriffs an einer Blutung verstarb. Im gleichen Jahr wurden vier weitere Eingriffe bei Erwachsenen vorgenommen, die ebenfalls erfolglos blieben. Erst die am 17. März 1971 von Professor Jean-Bernard Otte im belgischen Leuven an einem 17 Monate alten Kind vorgenommene Lebertransplantation verlief erfolgreich. Die Überlebensraten blieben jedoch sehr niedrig, bis in den achtziger Jahren Cyclosporin verfügbar wurde. Diese wirkungsvolle immunsuppressive Therapie ermöglichte es, die Transplantation solider Organe voranzutreiben. Später entwickelten sich die Chirurgietechniken mit der Transplantation von verkleinerten Lebern und Teillebern und der Alternative von Lebendspenden weiter.

Die Langzeit-Überlebensrate von Kindern, die sich einer Lebertransplantation unterziehen müssen, beträgt heute 95 Prozent.

In der Schweiz fand die erste Lebertransplantation bei einem Kind am 1.Juli 1989 im Rahmen eines ursprünglich für Erwachsene und Kinder vorgesehenen Programms zur Lebertransplantation im Genfer Universitätsspital (HUG) statt. Inzwischen hat sich dieses Programm auf Kinder spezialisiert, wobei weiterhin eine enge Zusammenarbeit mit dem Team für Erwachsene und eine nunmehr landesweit angelegte Kooperation mit einem medizinisch-chirurgischen und multidisziplinären Team gepflegt wird. In der Schweiz erfolgen somit alle pädiatrischen Lebertransplantatio- nen in einem einzigen Zentrum, im Schweizerischen Zentrum für Kinderleberkrankheiten der HUG. Bis heute wurden mehr als 170 pädiatrische Transplantationen durchgeführt; derzeit finden in Genf durchschnittlich zehn Transplantationen pro Jahr statt, was den Bedarf der Schweizer Bevölkerung abdeckt.

Noch vor 20 Jahren hatte eine Lebertransplantation vor allem das Überleben des Kindes als Ziel und war assoziiert mit einer Vielzahl an Komplikationen. Heute hingegen können wir die Familien beruhigen, da trotz der komplexen Behandlung das Ergebnis im Hinblick auf Lebensqualität und Lebenserwartung häufig hervorragend ist.

\section{Die bemerkenswerte Entwicklung der Lebertransplantation bei Kindern}

In der Schweiz unterscheiden sich die Indikationen für eine Lebertransplantation bei Kindern kaum von denen in der übrigen Welt: In 50\% der Fälle besteht die Ursache in einer Gallengang-Atresie. Einst lag die Überlebensrate der betroffenen Kinder nach 5 Jahren bei 30-55 Prozent, nach 10 Jahren bei 30 Prozent und nach 20 Jahren bei 20 Prozent. Dank der Lebertransplantationen nähern wir uns heute einer Überlebensrate von 100 Prozent. Kinder, die eine Lebertransplantation benötigen, sind meist jung und klein: Die Hälfte von ihnen wiegt zum Zeitpunkt der Transplantation weniger als 10 Kilogramm, zwei Drittel wiegen unter 15 Kilogramm. Die ersten pädiatrischen Lebertransplantationen dauerten noch rund 12 Stunden; heute ist die Dauer dank der Erfahrung des Chirurgie- und Anästhesieteams auf die Hälfte gesunken. Auch der Spitalaufenthalt verkürzte sich immer weiter auf mittlerweile 
zwei bis vier Wochen, während die Kinder in den neunziger Jahren noch sehr lange im Krankenhaus bleiben mussten, was für sie und ihre Familien sehr belastend und aufwendig war. Mortalität und Morbidität der Lebertransplantation bei Kindern hängen mit chirurgischen Aspekten (Blutungen, Thrombosen, Gallenkomplikationen usw.) und medizinischen Aspekten (primäre Nichtfunktion, Infektion usw.) zusammen. Diese treten am häufigsten im ersten Jahr nach der Transplantation auf und erfordern sehr spezielles Know-how. Die allgemeine Überlebensrate von Kindern nach einer Lebertransplantation hat sich in Genf erstaunlich entwickelt: Lag die Mortalität während der ersten fünf Jahre des Genfer Programms noch bei 13 Prozent, so beträgt sie heute (zwischen 2011 und 2016) nur noch 5 Prozent. Beim Überleben des Transplantats ist die Entwicklung mit einem Anstieg von 72 Prozent (1989-1994) auf 89 Prozent (2011-2016) noch beeindruckender.

\section{Das eigentliche Ziel: Entwicklung und Lebensqualität}

Anfangs sollten Lebertransplantationen kranken Kindern primär das Überleben ermöglichen. Heute steht über den Aspekt des Überlebens hinaus die weitgehende Normalisierung der Lebensqualität der Patienten im Vordergrund und das Bestreben, keine chroni-

\section{Normalerweise können die Kinder einige Wochen nach der Transplantation wieder zur Schule gehen, Sport treiben, in den meisten Fällen später den Beruf ihrer Wahl erlernen und selbst Kinder bekommen.}

Korrespondenz: Prof. Barbara Wildhaber Centre Universitaire Romand de Chirurgie Pédiatrique Service de chirurgie pédiatrique Hôpital des Enfants Hôpitaux Universitaires de Genève Rue Willy Donzé 6

CH-1205 Genf

Tel. 0223724662 Fax 0223724666 Barbara.Wildhaber[at] hcuge.ch sche Erkrankung durch eine andere zu ersetzen. Die Patienten sollen wie gesunde Kinder leben können, einige Wochen nach der Transplantation wieder zur Schule gehen, Untersuchungen nicht aufwendig stationär, sondern ambulant durchführen lassen, mit ihren Freunden in Ferienlager fahren, in den meisten Fällen den Beruf ihrer Wahl erlernen und eine Familie gründen können. Die Teams, die diese Kinder begleiten, müssen darum multidisziplinär, spezialisiert und kompetent sein, den Kindern und Jugendlichen optimale Überlebenschancen mit einer hervorragenden
Lebensqualität bieten zu können, auch wenn die $\mathrm{Pa}$ tienten auf Dauer Medikamente einnehmen müssen, die allerdings nicht sehr belastend sind.

Was die psychomotorische und/oder intellektuelle Entwicklung betrifft, bleiben die schulischen Leistungen von transplantierten Patienten leicht unterdurchschnittlich, was wahrscheinlich auf die Erkrankung zurückzuführen ist, die die Transplantation erforderlich gemacht hat. Nichtsdestotrotz ist die Integration in die Gesellschaft bei dieser Patienten im Erwachsenenalter sowohl in beruflicher als auch privater Hinsicht sehr befriedigend. Bestes Beispiel für diese Entwicklung ist die Möglichkeit, Kinder zu haben.

Zusammenfassend verdeutlicht die Lebertransplantation bei Kindern bestens, wie das Zusammenspiel von äusserst spezialisierter Medizin und Multidisziplinarität nicht nur die Überlebensrate der Patienten erhöht, sondern auch ihre Lebensqualität und ihre Zukunft als Bürger unseres Landes sicherstellt.

\section{Zusammenfassung}

Bei terminalen chronischen und akuten Lebererkrankungen von Kindern ist die Lebertransplantation die Behandlung der Wahl. Dieser äusserst komplexe medizinisch-chirurgische Eingriff mit extrem hohen Ansprüchen im Hinblick auf Personal und Medizintechnik wurde wegen seiner Mortalität und Morbidität oft kritisiert. Die Überlebensrate von Kindern nach einer Lebertransplantation hat sich allerdings erstaunlich entwickelt: Lag die Mortalität innerhalb der ersten fünf Jahre des Genfer Programms noch bei 13 Prozent, so beträgt dieser Wert heute nur noch 5 Prozent, das Überleben des Transplantats stieg von 72 auf 89 Prozent. Heute können die jungen Patienten nahezu wie gesunde Kinder leben und profitieren auch im Erwachsenenalter von einer guten beruflichen wie privaten Lebensqualität. Die Lebertransplantation bei Kindern ist damit ein sehr gutes Beispiel dafür, wie hochspezialisierte Medizin und eine multidisziplinäre Zusammenarbeit nicht nur die Überlebensraten der Patienten erhöhen, sondern auch ihre Lebensqualität und ihre Zukunft als Bürger unseres Landes sicherstellen.

\section{Dank}

Die Autoren danken Simona Korff, PhD, für die Führung des Schweizer Kinderlebertransplantationsregisters und die Berechnung lokaler Statistiken. 